\title{
ELECTROSPUN THERMOPLASTIC POLYURETHANE MATS CONTAINING NAPROXEN- CYCLODEXTRIN INCLUSION COMPLEX
}

\author{
Çiğdem Akduman', Işık Özgüney², E. Perrin Akçakoca Kumbasar ${ }^{3}$ \\ ${ }_{1}$ Pamukkale University, Denizli Vocational School of Technical Sciences, Department of Textile Technology, Denizli, Turkey \\ ${ }^{2}$ Ege University, Faculty of Pharmacy, Department of Pharmaceutical Technology, Izmir, Turkey \\ ${ }^{3}$ Ege University, Faculty of Engineering, Department of Textile Engineering, Izmir, Turkey \\ E-mail: cakduman@pau.edu.tr
}

\begin{abstract}
:
Incorporation of cyclodextrins (CDs) into electrospun nanofibrous materials can be considered as potential candidates for functional medical textile applications. Naproxen (NAP) is a type of non-steroidal anti-inflammatory drug commonly administered for the treatment of pain, inflammation and fever. Drug-inclusion complex formation with $C D s$ is an approach to improve the aqueous solubility via molecular encapsulation of the drug within the cavity of the more soluble CD molecule. In this study, NAP or different NAP-CD inclusion complexes loaded nanofibres were successfully produced through electrospinning and characterised. The inclusion complex loaded mats exhibited significantly faster release profiles than NAP-loaded thermoplastic polyurethane (TPU) mats. Overall, NAP-inclusion complex loaded TPU electrospun nanofibres could be used as drug delivery systems for acute pain treatments since they possess a highly porous structure that can release the drug immediately.
\end{abstract}

\section{Keywords:}

Naproxen, $\beta$-cyclodextrin, 2-hydroxy propyl- $\beta-C D$, Inclusion complex, Electrospinning, Drug release

\section{Introduction}

Textile-based nonwoven drug delivery systems present unique properties, including high surface area, and drug loading capacity, fabrication ease and options for surface modifications make them attractive alternatives to current drug delivery devices [26]. The principle of electrospinning process is to use electrostatic force as the main driving power for fibre formation [24]. One of the obvious advantages of the electrospinning process is the highly porous structure of electrospun fibre mats, which exhibits much greater surface area and possibility of functionalization $[13,25]$. More specifically, incorporation of cyclodextrins (CDs) into electrospun nanofibrous materials is considered as potential candidates for functional textile applications [14].

Topical administration of active pharmaceutical substances is in principle favourable for rapid onset of drug action and ready accessibility [12]. This administration could be very efficient and useful in the treatment of acute pain related conditions [26]. The clinical efficacy of a topically applied drug depends not only on its pharmacologic properties but also on the drug availability at the target site [15]. Theoretically, topically applied NSAIDs could act directly within the area of injury, without systemic activity, and reduce the inflammatory reaction [8].

Naproxen (NAP) (Fig. 1a) is a propionic acid derivative and is a type of non-steroidal anti-inflammatory drug commonly administered for the treatment of pain, inflammation and fever $[9,11]$.

CDs are one of the most promising materials for the development of products with advanced properties due to inclusion complex formation ability with a wide variety of substances [16]. CDs have been explored extensively as additives in the pharmaceutical industry due to their ability to host and solubilize hydrophobic<smiles>COC(=O)[C@H](C)c1ccc2cc(OC)ccc2c1</smiles>

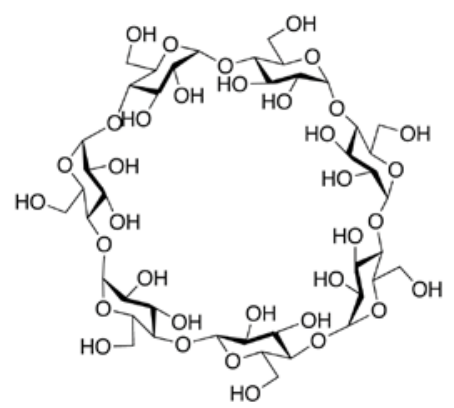

(a) (b)

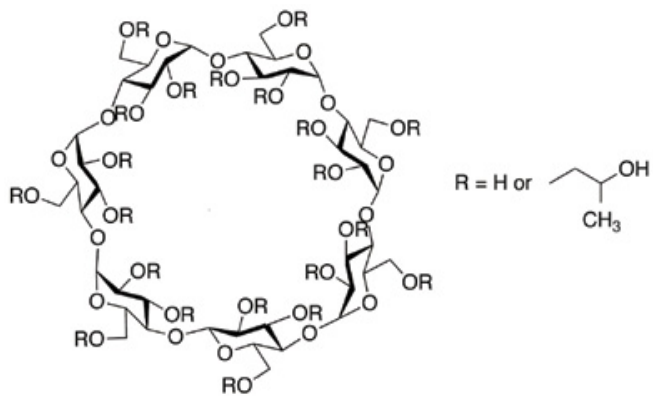

(c)

Figure 1. Naproxen (NAP) (a), $\beta-C D(b)$ and HP- $\beta-C D(c)[18,19]$. 
Table 1. Molecular weight and solubility parameters of $\beta$-cyclodextrin and 2 -hydroxy propyl- $\beta$-cyclodextrin [4]

\begin{tabular}{|c|c|c|}
\hline Cyclodextrin & Molecular weight (MW) (Da) & Solubility in water (mg/ml) \\
\hline$\beta$-Cyclodextrin ( $\beta-C D)$ & 1135 & $>6.5$ \\
\hline 2-Hydroxypropyl- $\beta$-cyclodextrin (HP- $\beta-C D)$ & 1400 & $>600$ \\
\hline
\end{tabular}

guest molecules including drugs $[16,23] . \beta-C D$, which is illustrated in Fig. 1b, is the most commonly used CD due to the cavity size, availability and low cost [5]. Apart from parent CDs its alkylated derivatives, e.g. 2-hydroxy propyl- $\beta-C D(H P-\beta-C D)$ (Fig $1 \mathrm{c})$, have also attracted growing interest due to their improved complexation efficiency, greater water solubility and less toxicity [3]. Inclusion complex formation with CDs is an approach to improve the aqueous solubility via molecular encapsulation of the drug within the cavity of the more soluble CD molecule [2, 21]. Molecular weight and solubility parameters of $\beta-C D$ and HP$\beta-C D$ were given in Table 1.

The objective of this study was to develop and characterize a novel electrospun nonwoven material loaded with NAP-CD inclusion complexes to obtain immediate release due to higher solubility enhancement of NAP by $\beta-C D$ and HP- $\beta-C D$ inclusion complex formation for topical treatment of pain-associated conditions with acute minor injuries. In this scope, NAP-CD inclusion complexes were prepared (Fig 2) and incorporated into thermoplastic polyurethane (TPU) nanofibrous mats via electrospinning process.

\section{Experimental}

\subsection{Materials}

TPU (Pellethane 2103-80AE, based on 4,4-methylene bisphenylene isocyanate, polytetramethyleneoxide and 1,4 butanediol) was received from Velox (Lubrizol Advanced Materials). NAP, N,N-dimethylformamide ( $\geq 99 \%)$, potassium dihydrogen phosphate(98-100.5\%) and sodium hydroxide $(\mathrm{NaOH})(99.99 \%)$ were purchased from Sigma-Aldrich. $\beta-\mathrm{CD}$ and HP- $\beta-C D$ were purchased from Wacker Chemie AG.

\subsection{The preparation of solid $N A P / \beta-C D$ and $H P \beta-C D$ inclusion complex}

NAP and CDs at a molar ratio of 1:1 were dissolved in enough equal volume of ethanol and ultra pure water, respectively, and then the two solutions were mixed and stirred at room temperature for 1 hour. Dried complexes were obtained by lyophilisation in a freeze dryer, where samples were frozen at $-80^{\circ} \mathrm{C}$ for approximately $48 \mathrm{~h}$ followed by drying at $0.05 \mathrm{mbar}$ for $24 \mathrm{~h}$. The powders obtained were stored in gas-tight bottles at room temperature until use.

\subsection{X-ray diffraction (XRD)}

$X$-ray powder diffraction patterns were determined for pure drug, $\beta-C D, H P-\beta-C D, N A P / \beta-C D$ and NAP/HP- $\beta-C D$ inclusion complexes. XRD patterns were recorded on Philips X'Pert Pro powder $\mathrm{X}$-ray diffractometer using $\mathrm{Cu} \mathrm{Ka}$ radiation for $2 \theta$ from 5 to $50^{\circ}$.

\subsection{Fourier transform infrared spectroscopy (FT-IR)}

FT-IR transmission spectra were recorded using a PerkinElmer FTIR spectrometer. Pure drug, $\beta-C D, H P-\beta-C D, N A P / \beta-$ $C D$ and NAP/HP- $\beta-C D$ inclusion complexes were previously mixed thoroughly with potassium bromide separately. Scans were obtained in a spectral range from 650 to $4000 \mathrm{~cm}^{1}$ with a resolution of $1 \mathrm{~cm}^{1}$.

\subsection{The preparation of electrospinning solutions}

$10 \%(w / w)$ of TPU stock solutions was prepared by dissolving TPU granules in N,N dimethylformamide at room temperature. The NAP, NAP/ $\beta-C D$ and NAP/HP- $\beta-C D$ loaded TPU solutions were obtained by dispersing the NAP and $C D$ inclusion complex powders in stock solutions. NAP concentrations were fixed at $10 \%$ with respect to polymer weight for all solutions. The weight of the inclusion complexes were calculated based on the amount of NAP $\left(10 \% \mathrm{w} / \mathrm{w}_{\text {polymer }}\right)$.

\subsection{Electrospinning}

Electrospinning of the polymer solutions was carried out by a set-up consisting of a syringe $(10 \mathrm{ml})$ and needle $(2.5-\mathrm{cm}$ long, 22 gauges, flat tip), a ground electrode and a high-voltage supply (Simco, MP Series CM5 30 P, Charging Generator Output $30 \mathrm{kV} \mathrm{DC}$ ). Polymer solutions were electrospun at a voltage of $13 \mathrm{kV}$, and a tip-to-collector distance of $20 \mathrm{~cm}$ with a feeding rate of $1 \mathrm{ml} / \mathrm{h}$. A grounded stationary rectangular metal collector covered with a piece of aluminium foil was used for the nanofibre deposition.

\subsection{Scanning electron microscopy (SEM) and thickness measurements}

The morphology of NAP, $\beta-C D, H P-\beta-C D, N A P / \beta-C D$ and NAP/HP- $\beta-C D$ inclusion complexes and the electrospun TPU nanofibres was characterized using SEM (FEI Quanta 250FEG). Each sample was coated with a thin film of gold using an EMITECH K550X ion sputtering device. Fibre diameters were determined by using Image $J$ software. Fibre diameters were measured by using SEM images by drawing straight lines perpendicular to the fibre axis. The pixel values of the lines were converted to diameter values. Fifty measurements were carried out from the different parts of each sample. The thickness of the nanofibrous mats was measured at eight points by a Mitutoyo digital micrometer at 
$0.001 \mathrm{~mm}$ accuracy and average thickness is calculated from these measurements.

\subsection{Statistical analysis}

All results were expressed as mean $\pm S D$. Data were analysed by one-way ANOVA followed by Tukey HSD post hoc test. Differences of $P<0.05$ were considered statistically significant.

\subsection{In vitro drug release studies}

Total immersion method was used to study the release characteristics of the drug from the NAP-loaded electrospun TPU fibre mats. The samples (in dimension of $3 \times 3 \mathrm{~cm}$ ) were accurately weighed and immersed in a glass bottle containing phosphate buffer $\mathrm{pH} 7.4 \mathrm{USP}$ and the bottles were incubated in a horizontal incubation shaker (Thermo-Scientific MaxQ6000) at $37 \pm 0.5^{\circ} \mathrm{C}$ and $60 \mathrm{rpm}$. At a specified immersion period ranging between 0 and $120 \mathrm{~h}$ ( 5 days), $0.5 \mathrm{ml}$ of the sample was taken out at selected times and an equal volume of medium was returned to the system after withdrawal. The samples were then assayed spectrophotometrically at 263 $\mathrm{nm}$. The experiments were carried out in triplicate. The release of the drug from the samples was reported as the cumulative release of the drug as a function of the immersion period. The calibration curve of NAP was prepared by using stock solutions in 22 different concentrations between 0.625 and 50 $\mu \mathrm{g} / \mathrm{ml}$. It showed linearity and acceptability with $R^{2} \geq 0.99$. The maximum absorbance measurement results were adapted to this calibration curve.

\section{Results and discussion}

\subsection{X-ray diffraction (XRD)}

The XRD patterns of the pure NAP, pure $\beta-C D$ and HP- $\beta-C D$, $N A P / \beta-C D$ and NAP/HP- $\beta-C D$ inclusion complexes were recorded to investigate the possible differences in the crystallinity of the structures (Fig. 2). In the X-ray diffractogram of pure NAP powder (Fig. 2e), sharp peaks at a diffraction angle of $2 \theta 6.5$, $12.5,13,16.5,18,19,20,22,23.5,27$ and 28.2 are present, and it suggests that the drug is present as a crystalline material [1]. The X-ray diffractogram of NAP/HP- $\beta$-CD powder (Fig.2c) showed with respect to the diffraction patterns of the starting materials, the broadening, disappearance and intensity diminution of some NAP diffraction peaks, especially those situated at 7,19 and $29^{\circ}$ $(-2 \theta)$. These findings suggest the presence of a new solid phase with a lower degree of crystallinity, which could be originated by the molecular interaction of the host HP- $\beta-C D$ and the guest NAP. NAP/ $\beta-C D$ (Fig. 2a) presented a diffraction pattern completely diffused, with the disappearance of the characteristic peaks of NAP and $\beta-C D$, reflecting the amorphous nature of this binary system. This is a direct proof of the formation of a new solid phase and can be considered as a very probable indication of the inclusion complex formation between NAP and $\beta-C D$ in the solid state [7].

\subsection{Fourier transform infrared spectroscopy (FT-IR)}

The FT-IR spectra of NAP-CD complexes were compared with the pure drug, $\beta-C D$ and HP- $\beta-C D$ powder. NAP peaks

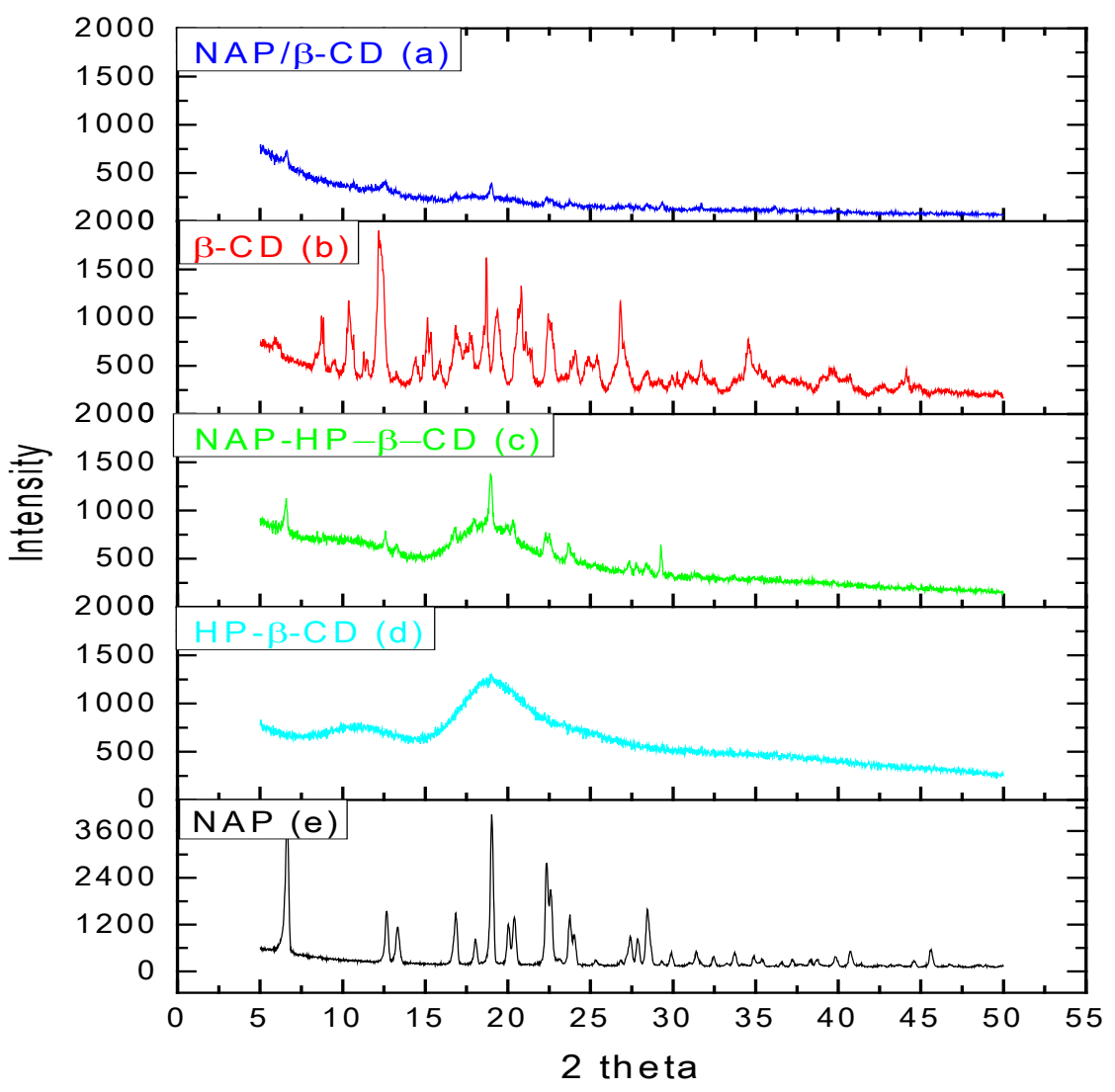

Figure 2. XRD diffraction patterns of (a) NAP/ $\beta-C D$ inclusion complex (b) $\beta-C D$, (c) NAP/HP- $\beta-C D$ inclusion complex, (d) HP- $\beta-C D$ and (e) naproxen (NAP). 
observed in region of $800-2000 \mathrm{~cm}^{-1}$ includes peaks at 1729 , indicating the $-\mathrm{C}=\mathrm{O}$ (carbonyl) stretch, peak at $1630 \mathrm{~cm}^{-1}$ due to $v(C-C)$ modes of the aromatic groups [6] and the peak at $1228 \mathrm{~cm}^{-1}$, indicative of the $-\mathrm{O}-$ stretch $[9,11,17]$ and 1394 $\mathrm{cm}^{-1}$ due to $\mathrm{CH}_{3}$ bending [10]. The vibration modes detected at 1026 and $857 \mathrm{~cm}^{-1}$ correspond to $\mathrm{C}-\mathrm{O}-\mathrm{C}$ bonds in NAP [27]. In the case of inclusion complexes, in particular, the characteristic carbonyl-stretching band of drug appeared at 1729 and shifted to $1728 \mathrm{~cm}^{-1}$ for NAP/ $\beta-C D$ and NAP/HP- $\beta-C D$ complexes, respectively, along with significantly reduced intensity of the same band. Characteristic absorption bands of the pure drug at $1604 \mathrm{~cm}$ [20] are maintained in NAP/ $\beta-C D$ with an evident reduction in intensity. Changes in the characteristic bands of pure drug confirm the existence of the complex as a new compound with different spectroscopic bands [22]

\subsection{Scanning-electron microscope (SEM) and thickness measurements}

Figure $4 a-e$ presents the SEM images of NAP, $\beta-C D, H P-\beta$ $C D$ and the corresponding $1: 1$ NAP/ $\beta-C D, N A P / H P-\beta-C D$ inclusion complexes, respectively. The SEM of NAP appeared as of distorted tubular shapes and sizes, and tended to form aggregates (Fig. 2a). HP- $\beta-C D$ is observed as "shrinked" cylindrical spheres (Fig. 2c), whereas $\beta-C D$ appeared as irregularly shaped crystals (Fig. 2b). In contrast, it was not possible to recognize individual component particles in the SEM image of the NAP/ $\beta-C D$ and NAP/HP- $\beta-C D$ complex (Fig. $2 d-e)$, thus indicating effective interaction between NAP and $\beta-C D$ and HP- $\beta-C D$. Therefore, SEM results, along with those of FT-IR and XRD, confirm the presence of inclusion complex in the solid state.
The morphology of electrospun nanofibres of pure NAP loaded, NAP/ $\beta-C D$ and NAP/HP- $\beta-C D$ inclusion complex loaded TPU nanofibres was investigated by SEM imaging. The representative images of SEM micrographs are given in Fig. 5. Based on the SEM images (Fig. $5 \mathrm{a}-\mathrm{C}$ ), bead-free nanofibres with diameter variation were obtained from all three TPUbased nanofibrous samples. NAP-loaded nanofibres were more uniform compared with the NAP/ $\beta-C D$ and NAP/HP- $\beta$ $C D$ inclusion complex loaded TPU nanofibres. Especially, there are several slubs along the NAP/ $\beta-C D$ inclusion complex loaded nanofibres axis. We hypothesize that these slubs would be preferably the result of the incorporation of the inclusion complexes to the fibres. After release tests, SEM images of $N A P / \beta-C D$ and NAP/HP- $\beta-C D$ inclusion complex loaded TPU nanofibres were completely different from their initial state. Complete disappearance of the fibre uniformity and roughening defects of these irregular fibres was also possibly associated with the dissolution of the inclusion complexes, which were primarily incorporated into the fibre axis. This result also supports the hypothesis of the incorporation of the inclusion complexes to the fibres.

Mean diameter of the fibres and mean thickness of the electrospun mats were summarized in Table 2. There was a statistically significant difference between the mean diameters of three groups as determined by one-way $\operatorname{ANOVA}(F(2,147)=$ $8.292, p=.000$ ). A Tukey post hoc test revealed that the mean diameter of the TPU nanofibres was statistically significantly higher after loading NAP/ $\beta-C D(879.68 \pm 159.12 \mathrm{~nm}, p=0.000)$ or NAP/HP- $\beta-C D(834.34 \pm 215.37 \mathrm{~nm}, p=0.016)$ inclusion complexes compared with the pure NAP loading (723.50 \pm $212.32 \mathrm{~nm}$ ). There were no statistically significant differences

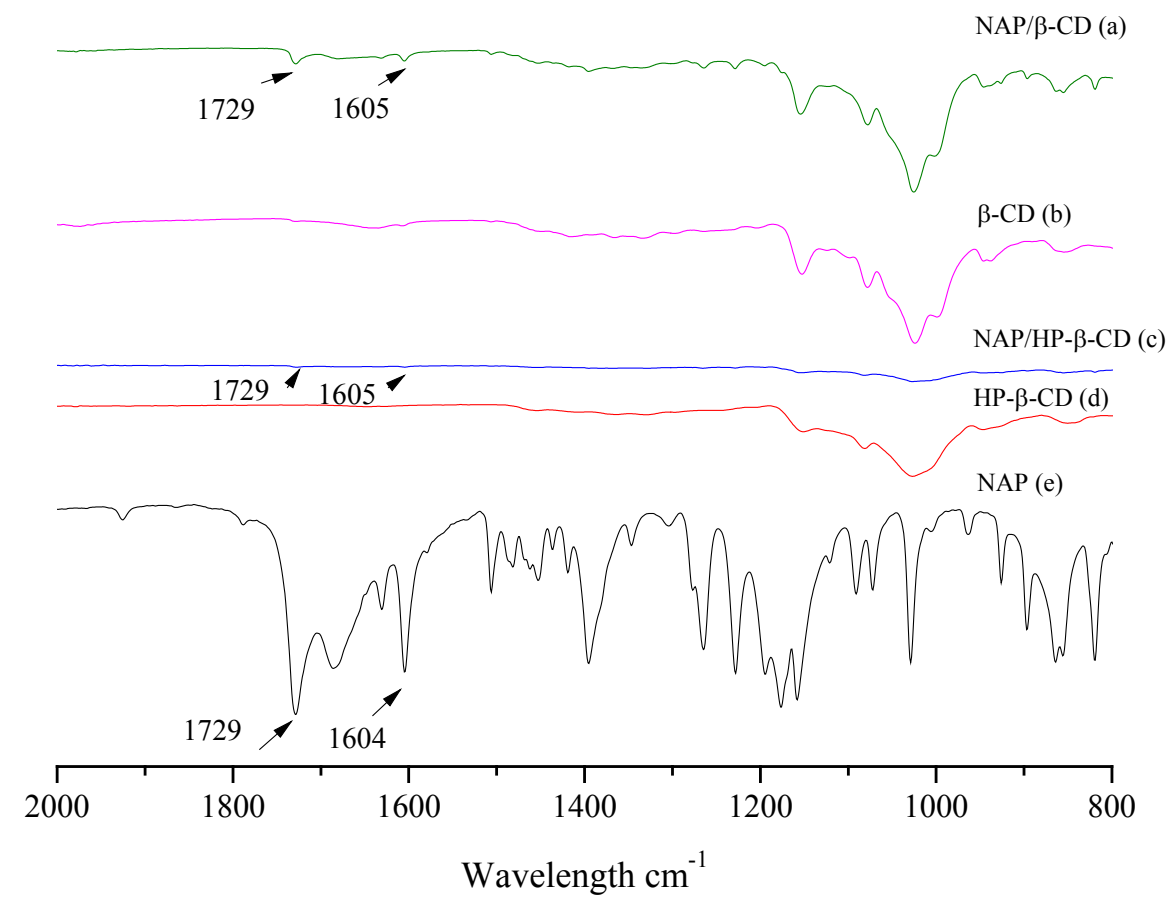

Figure 3. FTIR spectra of (a) NAP/ $\beta-C D$ inclusion complex (b) $\beta-C D$, (c) NAP/HP- $\beta-C D$ inclusion complex, (d) HP- $\beta-C D$ and (e) naproxen (NAP). 
Table 2. Mean diameter and mean thickness of NAP-TPU, NAP/ $\beta-C D-T P U$ and NAP/HP- $\beta-C D-T P U$ nanofibres and mats.

\begin{tabular}{|c|c|c|}
\hline & Mean diameter \pm SD & Mean thickness \pm SD \\
\hline NAP-TPU & $723.50 \pm 212.32$ & $0.77 \pm 0.22$ \\
\hline NAP/R-CD-TPU & $879.68 \pm 159.12^{*}$ & $1.37 \pm 0.37$ \\
\hline NAP/HP- $\beta-C D-T P U$ & $834.34 \pm 215.37^{*}$ & $1.05 \pm 0.13$ \\
\hline
\end{tabular}

${ }^{*} \mathrm{P}<0.05$. One-w-ay ANOVA, Tukey HSD post hoc test.

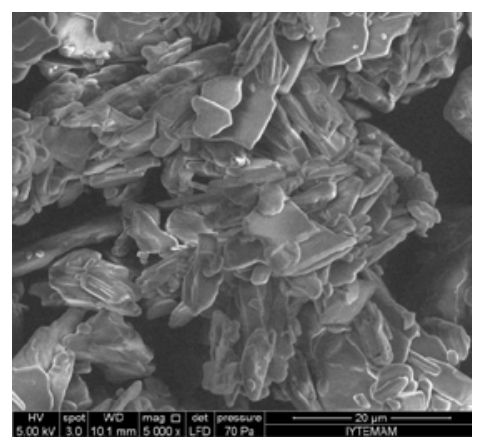

(a)

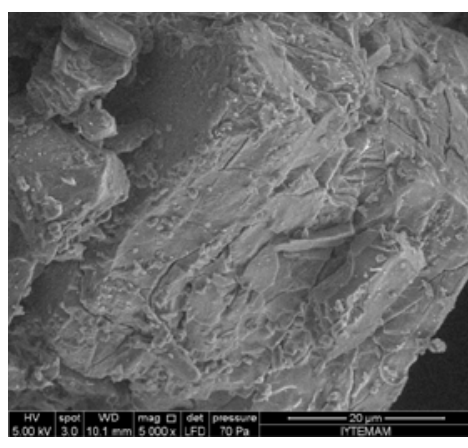

(b)

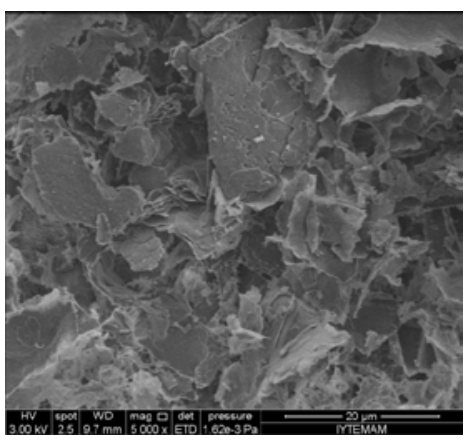

(d)

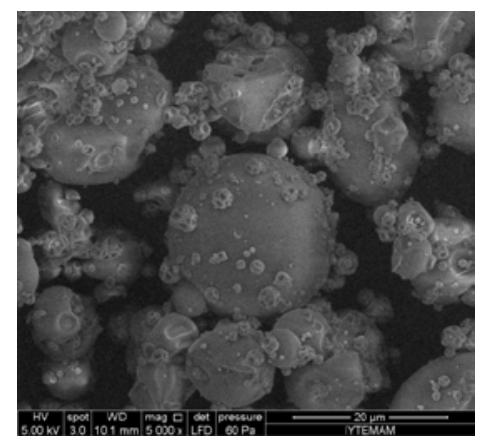

(c)

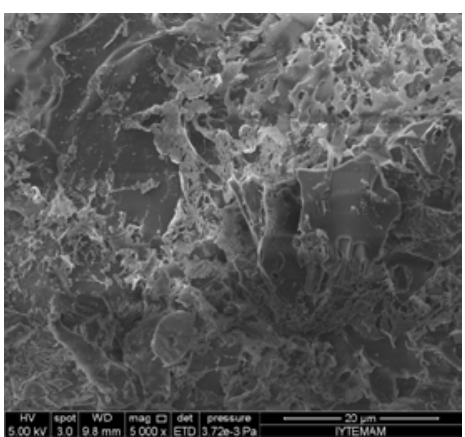

(e)

Figure 4. SEM images of (a) naproxen (NAP), (b) $\beta-C D$, (c) HP- $\beta-C D$, (d) NAP/ $\beta-C D$ inclusion complex and (e) NAP/HP- $\beta-C D$ inclusion complex.

between the diameters of NAP/B-CD and NAP/HP- $\beta-C D$ groups $(p=0.486)$. In case of mean thickness, similar to mean diameters, there was a significant effect of NAP, NAP/ $\beta-C D$ or NAP/HP- $\beta-C D$ loading on the mat thickness at $p<0.05$ level of the three conditions $[F(2,69)=32.34, p=0.000)]$.

\subsection{In vitro drug release studies}

The release characteristics of NAP from NAP, NAP/ $\beta-C D$ and NAP/HP- $\beta-C D$ inclusion complex loaded electrospun TPU mats are shown in Fig. $6 \mathrm{a}-\mathrm{c}$. The release rates were investigated in PBS of $\mathrm{pH} 7.4$ at $37^{\circ} \mathrm{C}$. Pure NAP loaded fibres released about $54 \%$ of its drug content within 24 hours and reached about $94 \%$ of its drug content within 96 hours (4 days). NAP/ HP- $\beta-C D$ inclusion complex loaded fibres showed the fastest release compared with the NAP/ $\beta-C D$ inclusion complex and NAP-loaded fibres. It released about $50 \%$ of its NAP content within $30-45$ minutes, $80 \%$ of its NAP content between 1 and 1.5 hour and reached about $98 \%$ of its NAP content after 4 hours, whereas NAP/ $\beta-C D$ inclusion complex loaded fibres released about $50 \%$ of its NAP content between 1.5 and 2 hour, $80 \%$ of NAP content after 8 hour and completed its release after 30 hours. The release profiles revealed that NAP/ HP- $\beta-C D$ inclusion complex loaded TPU nanofibrous system has more than two times higher release rate than NAP/ $\beta-C D$ inclusion complex loaded TPU system. NAP/ $\beta-C D-T P U$ system has more than two times higher release rates than pure NAP loaded TPU system. As hydrophilic CDs can modify the rate of drug release [22], in this study it was shown that drug release rates from electrospun fibrous systems can be modified by using $\beta-C D$ or HP- $\beta-C D$ drug inclusion complex formation. To show the release characteristics of NAP or NAP-CD complex loaded electrospun nanofibrous systems, the release profiles for 8,48 and 120 hours were given separately.

\section{Conclusions}

NAP and its $\beta-C D, H P-\beta-C D$ inclusion complexes were successfully prepared by freeze-drying process. Prepared $N A P / \beta-C D$ and NAP/HP- $\beta-C D$ inclusion complexes were loaded to electrospun TPU fibres separately and their release characteristics were compared with pure NAP loaded TPU fibres. NAP/ $\beta-C D$ and NAP/HP- $\beta-C D$ inclusion complexes 
(a)

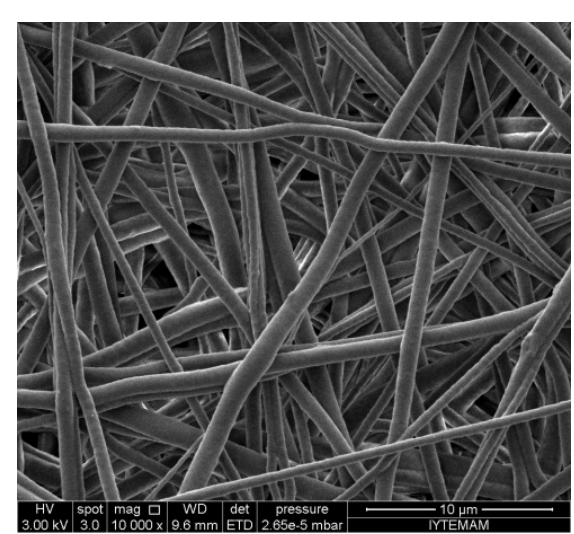

(b)

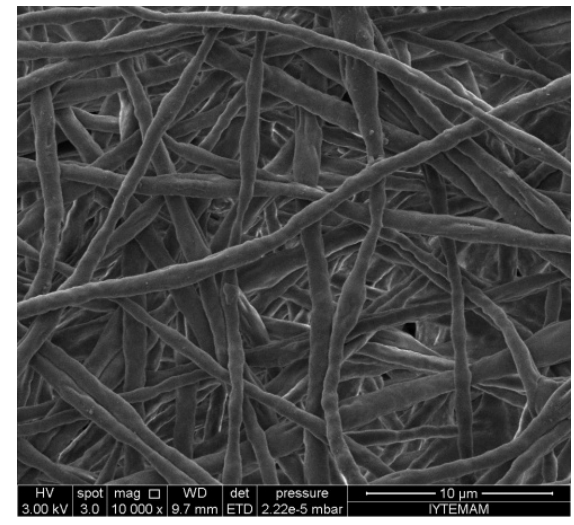

(c)

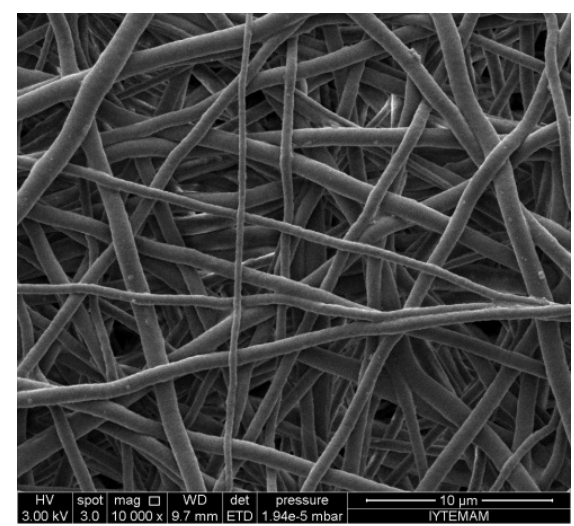

(d)

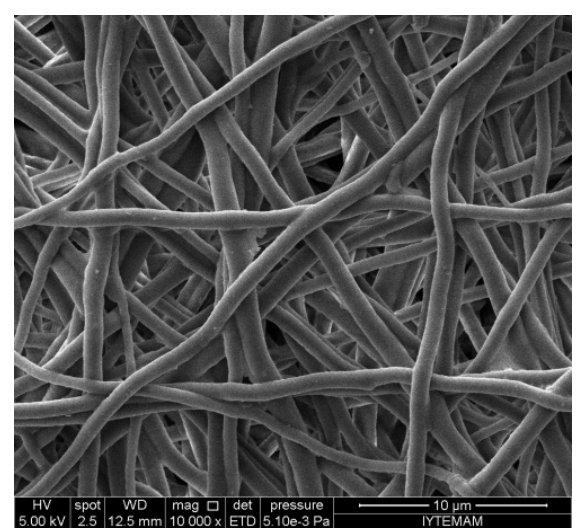

(e)

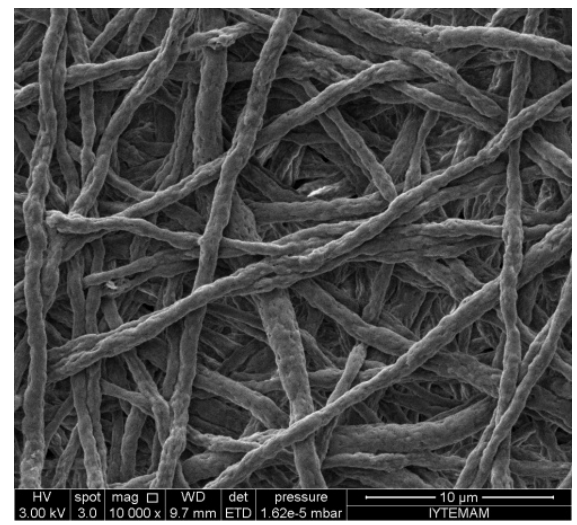

(f)

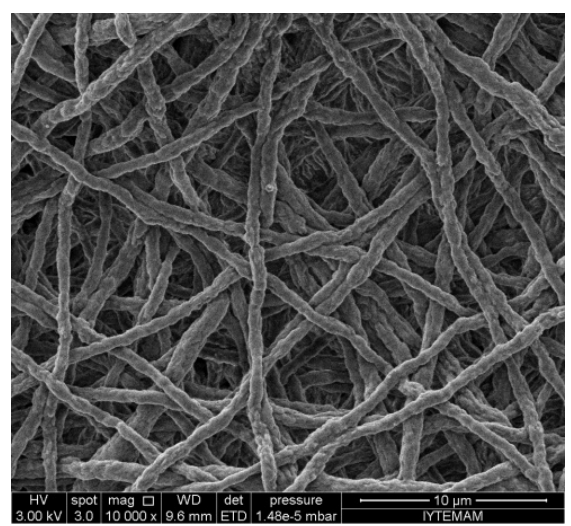

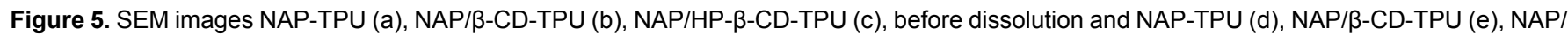
HP- $\beta$-CD-TPU (f) after dissolution.

loading have a significant effect on the resultant fibre diameter when compared with the pure NAP loading. It was seen that NAP release rate can be increased by using CD complexes because of the interaction ability of $\beta-C D$ and HP- $\beta-C D$ with poorly-water soluble drugs to increase their apparent solubility [4]. Overall, combining highly porous structure of the electrospun fibres, NAP-inclusion complex loaded TPU electrospun nanofibres could be used as textile-based drug delivery systems for acute pain treatments that can release the drug immediately.

\section{Acknowledgements}

The authors acknowledge Research Foundation of Ege University for the financial support given to this study (Project number: 13 MUH 051) and to Scientific Research Projects of Pamukkale University for support given for symposium registration fee. The authors also thank "the Pharmaceutical Sciences Research Centre, Faculty of Pharmacy, Ege University". 
(a)

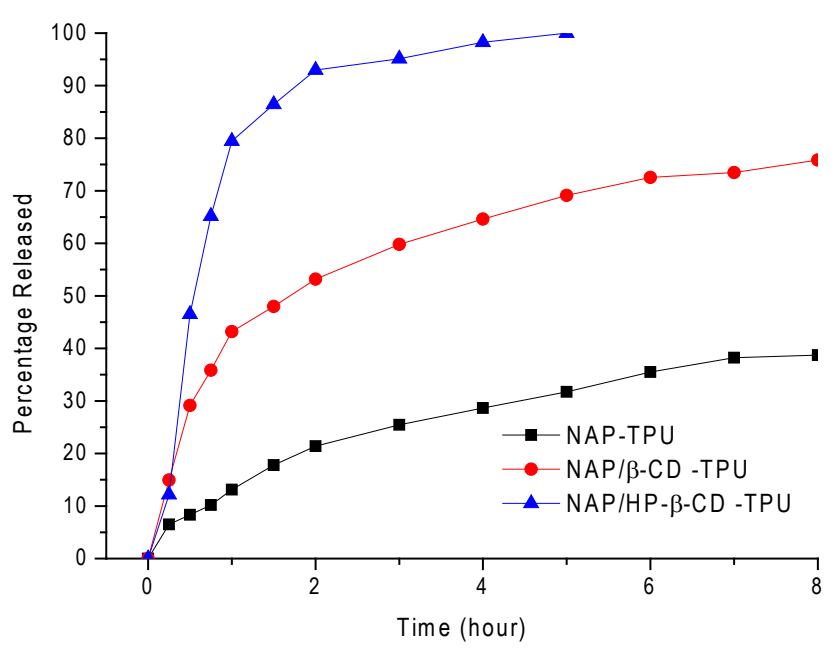

(b)

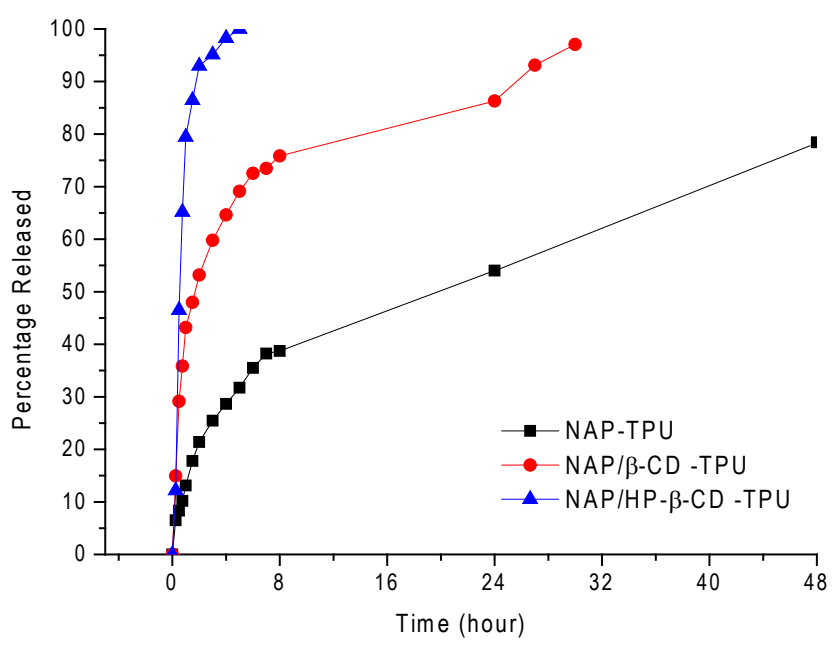

(c)

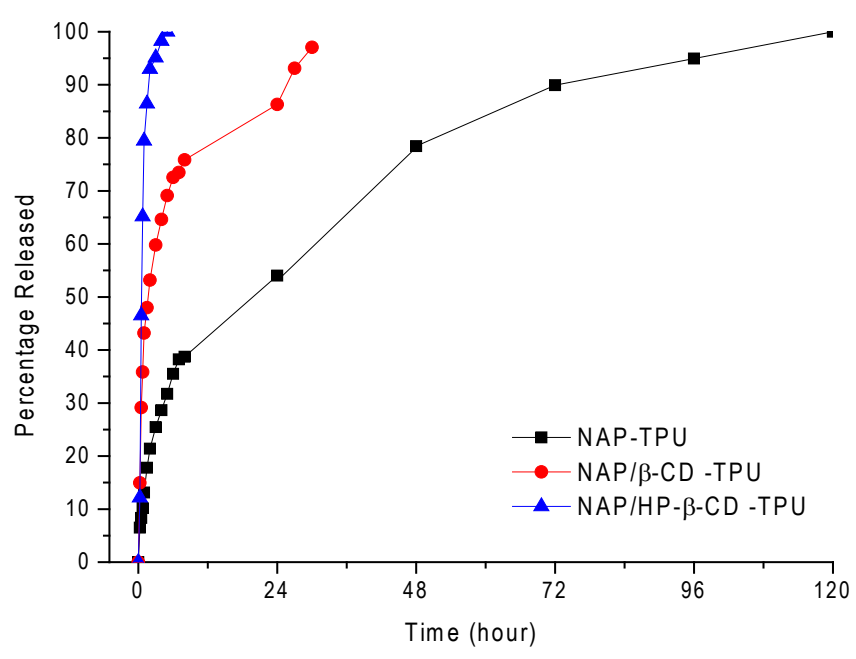

Figure 6. Percentage release of NAP from ( $\bullet$ NAP-TPU, $(\bullet)$ NAP/ $\beta-C D$ and $(\Delta)$ NAP/HP- $\beta-C D$ TPU mats by total immersion technique during $0-8$ hours (a), 0-48 hours and (b) 0-120 hours (c). 


\section{References}

[1] Adibkia, K., Barzegar-Jalali, M., Maheri-Esfanjani, H., Ghanbarzadeh, S., Shokri, J., Sabzevari, A., Javadzadeh, Y., (2013). Physicochemical characterization of naproxen solid dispersions prepared via spray drying technolog. Powder Technology. 246, 448-455.

[2] Ammar, H.O., Ghorab, M., Mahmoud, A.A., Makram, T.S., Noshi, S.H., (2012). Topical liquid crystalline gel containing lornoxicam/cyclodextrin complex. J Incl Phenom Macrocycl Chem. 73, 161-175.

[3] Banik, A., Gogoi, P., Saikia, M.D., (2012). Interaction of naproxen with $\beta$-cyclodextrin and its derivatives/polymer: experimental and molecular modeling studies. $J$ Incl Phenom Macrocycl Chem. 72, 449-458.

[4] Brewster, M. E., Loftsson, T., (2007). Cyclodextrins as pharmaceutical solubilizers. Advanced Drug Delivery Reviews. 59, 645-666.

[5] Del Valle, E.M., (2004). Cyclodextrins and their uses: a review, Process Biochemistry. 39, 1033-1046.

[6] Del Arco, M., Gutierrez, S., Martin, C., Rives, V., Rocha, J., (2004). Synthesis and characterization of layered double hydroxides (LDH) intercalated with non-steroidal anti-inflammatory drugs (NSAID). Journal of Solid State Chemistry. 177, 3954-3962.

[7] Fernandes, C.M., Veiga, F.J.B., (2002). Effect of the Hydrophobic Nature of Triacetyl- $\beta$-cyclodextrin on the Complexation with Nicardipine Hydrochloride: Physicochemical and Dissolution Properties of the Kneaded and Spray-dried Complexes. Chem. Pharm. Bull. 50(12), 1597-1602.

[8] Galer, B.S., Rowbotham, M., Perander, J., Devers, A., Friedman, E., (2000) Topical Diclofenac Patch Relieves Minor Sports Injury Pain: Results of a Multicenter Controlled Clinical Trial. Journal of Pain and Symptom Management. 19(4), 287-294.

[9] Grandelli, H.E., Stickle, B., Whittington, A., Kiran, E., (2013). Inclusion complex formation of $\beta$-cyclodextrin and Naproxen: a study on exothermic complex formation by differential scanning calorimetry. J Incl Phenom Macrocycl Chem. 77, 269-277.

[10] Javadzadeh, Y., Ahadia, F., Davarana, S., Mohammadi G., Sabzevarid, A., Adibkia, K., (2010). Preparation and physicochemical characterization of naproxen-PLGA nanoparticles. Colloids and Surfaces B: Biointerfaces. 81, 498-502.

[11] Junco, S., Casimiro, T., Ribeiro, N., Ponte, M.N., Marques, H.C., (2002). A Comparative Study of Naproxen - Beta Cyclodextrin Complexes Prepared by Conventional Methods and Using Supercritical Carbon Dioxide. Journal of Inclusion Phenomena and Macrocyclic Chemistry. 44, 117-121.
[12] Benson, H.A.E., Watkinson, A.C., (2012). Transdermal and Topical Drug Delivery: Principles and Practice (I ed.). John Wiley \& Sons Inc. (New Jersey).

[13] Kenawy, E., Abdel-Hay, F.I., El-Newehy, M.H., Wnek, G.E., (2007). Controlled release of ketoprofen from electrospun poly(vinyl alcohol) nanofibers. Materials Science and Engineering. 459, 390-396.

[14] Kumbasar, E.P.A., Akduman, C., Cay, A., (2014). Effects of $\beta$-cyclodextrin on selected properties of electrospunthermoplastic polyurethane nanofibres. Carbohydrate Polymers. 104, 42-49.

[15] Loftsson, T., (1998). Cyclodextrins: new drug delivery systems in dermatology, International Journal of Dermatology. 37, 241-246.

[16] Loftsson, T., Masson, M., (2001). Cyclodextrins in topical drug formulations: theory and practice. International Journal of Pharmaceutics. 225, 15-30.

[17] Machin, R., Isasi,J.R., Vélaz, I., (2012). $\beta$-Cyclodextrin hydrogels as potential drug delivery systems. Carbohydrate Polymers. 87, 2024- 2030.

[18] May 20, 2014. http://www.sigmaaldrich.com

[19] May 20, 2014. http://www.trc-canada.com/index.php

[20] Mura P., Faucci, M. T., Bettinetti G. P., (2001). The influence of polyvinylpyrrolidone on naproxen complexation with hydroxypropyl-b-cyclodextrin. European Journal of Pharmaceutical Sciences. 13, 187-194.

[21] Piao, Z., Lee, M., Lee, B., (2008). Colonic release and reduced intestinal tissue damage of coated tablets containing naproxen inclusion complex. International Journal of Pharmaceutics. 350, 205-211.

[22] Pralhad, T. and Rajendrakumar, K., (2004). Study of freeze-dried quercetin-cyclodextrin binary systems by DSC, FT-IR, X-ray diffraction and SEM analysis. Journal of Pharmaceutical and Biomedical Analysis. 34, 333-339.

[23] Szejtli, J., (1998). Introduction and General Overview of Cyclodextrin Chemistry, Chem. Rev. 98, 1743-1753.

[24] Subbiah, T., Bhat, G.S., Tock, R.W., Parameswaran, S., Ramkumar, S.S., (2005). Electrospinning of Nanofibers. Journal of Applied Polymer Science. 96, 557-569.

[25] Taepaiboon, P., Rungsardthong, U., Supaphol, P., (2006). Drug-loaded electrospun mats of poly(vinyl alcohol) fibres and their release characteristics of four model drugs. Nanotechnology, 17, 2317-2329.

[26] Bartels, V. T., (2011) Handbook of medical Textiles (I ed.). Woodhead Publishing Limited (Cambridge).

[27] Wei, M., Shi, S., Wang, Ji, Li, Y., Duan, X., (2004). Studies on the intercalation of naproxen into layered double hydroxide and its thermal decomposition by in situ FT-IR and in situ HT-XRD. Journal of Solid State Chemistry. 177, 2534-2541. 